\title{
Concept of an loT (Internet of Things)-based smart cloud service platform for agriculture
}

\author{
Yu Li \\ (Qingyuan Vocational Technical College, Qingyuan 511510, China)
}

Keywords: internet of things; intelligent agriculture; cloud platform; service

\begin{abstract}
The future of agriculture lies in standardized, large-scale, smart production of agricultural commodities. In light of growing adoption of IoT technologies in agriculture, this paper offers a description of the features of agricultural IoT, including its functional components, and proposes the framework of an IoT-based smart cloud service platform for agriculture.
\end{abstract}

\section{INTRODUCTION}

For the foreseeable future, developing modernized agriculture is an important task for the national and local governments of China. It is a mission made urgent by both the current state of China's first sector, and the reality of its rural economies, which need ways to optimize resource allocation, raise productivity, and build new models of production and management. Or as stated in 2017's No. 1 Central Government Document, a structural optimization of agricultural production is the foremost mission for rural regions. Information technologies have provided the perfect foundation for such a transformation. Observers have noted that the future of agriculture lies in standardized, large-scale, and smart production, in which resources are integrated by management information systems and artificial intelligence, commodities are produced with precision control, and uncertainties are eliminated. ${ }^{[1]}$ For example, sensory devices can be used to capture information from environments and work processes; such data can be mined with big data technologies; the results can be analyzed to achieve high-precision control, e.g. of fertilizers and pesticides. When each step is controlled with smart technologies, a new kind of energy and material efficient, zero-pollution agriculture can then be achieved.

\section{IOT APPLICATIONS IN AGRICULTURE}

\subsection{Context of IoT application in agriculture}

The digitization index study by McKinsey Global Institute showed agriculture to be the sector with the lowest degree of digitization. Most agricultural work processes in China are still done in traditional, small-scale, inefficient ways, even as a transformation is taking place towards digitally controlled precision agriculture. IoT technologies can play a part in this move towards industrialized production. The greatest problems facing China's agriculture include: first, agricultural commodities have short industrial chains where production, supply, and distribution have not been efficiently integrated, which keeps the profit of initial producers at an unsustainably low level; second, production is largely accomplished by traditional cultivation methods, and additionally, economies of scale are difficult to achieve due to land area restrictions; third, rural collectives and individual farmers severely lack the capital and resources required for reinvestment. For these reasons, it can be effective path to achieve the transformation by introducing modern IT to reconfigure traditional agricultural resources (water, land, consumables, machinery, labor, market and information) with new approaches of production and management.

On the national policies front, IoT has already been a focal point of the global technological race. The Chinese national government first named IoT as one of the emerging strategic fields of research to focus on in its 12th Five-Year Plan (2011 2015). For the 13th FYP (2016 2020), the Ministry of Agriculture issues policies to offer full support for agricultural IoT. On the technological front, China's agricultural IoT has continued to develop in recent years, particularly with progresses in 
organism detection, system development, and datamining; quality tracking technologies have been developed for real-time capturing and transmission, certification systems, pre-warning models, and end-product tracing; for logistics, category and coding standards have been established for electronic tags, and software platforms have been built for logistical information and e-commerce management. ${ }^{[2]}$

The IT field often considers IoT its fourth great wave after the invention of computers, the internet, and mobile networks. IoT (internet of things) builds upon the internet by using RFID and wireless communications to facilitate universal connections between all persons and objects. Inhuman objects (commodities) can directly communicate with each other without human intervention, as objects are identified by RFID, and information is shared through wireless channels. Agriculture is a sector where IoT has potential for many applications. With agricultural IoT, the environmental parameters, e.g. temperature, humidity, $\mathrm{pH}$, sunlight, soil nutrition and $\mathrm{CO} 2$ concentration, can be detected by IoT devices, and displayed as data on instruments, or be fed into the automated control system to maintain the most favorable conditions. ${ }^{[3]}$ With wireless networks, the technical staff will be able to control the environment of multiple greenhouses from their office, and control the productivity, quality and growth rate of the crops to maximize economical gain.

Agricultural IoT applications are already on the path to wide market adoption. An example is Adama Agricultural Solutions Co., Ltd., headquartered in Israel, which had merged with Hubei Sanonda Co., Ltd. to become the first transnational agricultural corporation listed in one of China's A-stock markets (the Shenzhen Stock Exchange). The company specializes in IoT solutions for monitoring pests. Their integrated system can capture photographic information of crops, which are stored in the cloud and analyzed to provide timely warnings to farmers on the dynamics of pest groups on both desktop and mobile devices, who can apply pest control with better preparations.

\subsection{Features of IoT applications in agriculture}

Despite the crucial role they will play, agricultural IoT technologies are still in an early exploratory stage. It is necessary to understand their features before predicting their future. Table 1. shows features gleamed from existing IoT applications

\begin{tabular}{lll}
\hline No. & \multicolumn{1}{c}{ Feature } & \multicolumn{1}{c}{ Description } \\
\hline 1 & Inter-connecti \\
& vity & $\begin{array}{l}\text { Inter-connectivity between persons, computers and objects, which is } \\
\text { even more important in agricultural IoT. Essential to optimization and } \\
\text { coordination of the three. }\end{array}$ \\
& Agricultural IoT focuses on organisms, and capturing \& transmitting \\
biological information is its core task. Precision control requires \\
analyzing and simulating relationships between organisms, and \\
explaining how they develop and change before decisions can be \\
made.
\end{tabular}




\section{FUNCTIONS OF AGRICULTURAL IOT}

\subsection{Datamining}

Sensory devices are used to capture and convert biological and environmental information, and transmitted to cloud services via mobile networks. The data are stored and analyzed at cloud services. Its components include sensor hardware, wireless gateways for connecting to the internet, high-performance data servers and massive storages, and data processing and visualization services. [5]

\subsection{Remote control}

Users can log into the remote system using desktop computers or mobile phones, and control air conditioning, pumps, ventilation and other devices in the cultivation environment. They may also design control schemas for the control system to execute, e.g. starting and stopping devices when environmental parameters reach the designated thresholds.

\subsection{Real-time lookup}

Users can look up environmental parameters in real time from their mobile devices, and perform statistical analysis on historical environmental and hardware parameters, and crop conditions. The system may also provide general information services, supplying farmers with the most up-to-date agricultural policies, market information, and supply-and-demand requests.

\subsection{Early warning}

Threshold values can be set on the required conditions for crop growth or storage, based on their type, lifecycle and seasonal variations. The system can issue timely warning to farmers when any of the thresholds are exceeded.

\section{LOGICAL FRAMEWORK FOR SMART CLOUD-BASED AGRICULTURAL SERVICES}

To achieve the acquisition and transmission of information on agricultural production, a smart cloud-based platform is necessary. Based on the highly scattered placements of agricultural resources, and the heterogeneous and complex nature of agricultural knowledge, we propose a framework for integrating agricultural resources and knowledge (Figure 1). As a solution to the dynamic allocation of resources, and the sharing of heterogeneous knowledge, it has two separate management centers to address their integration. ${ }^{[6]} \mathrm{A}$ portal website is used to coordinate the two centers and facilitate knowledge sharing, allowing users' knowledge and resources to integrate and interact. Its features are expounded in the following sections. 


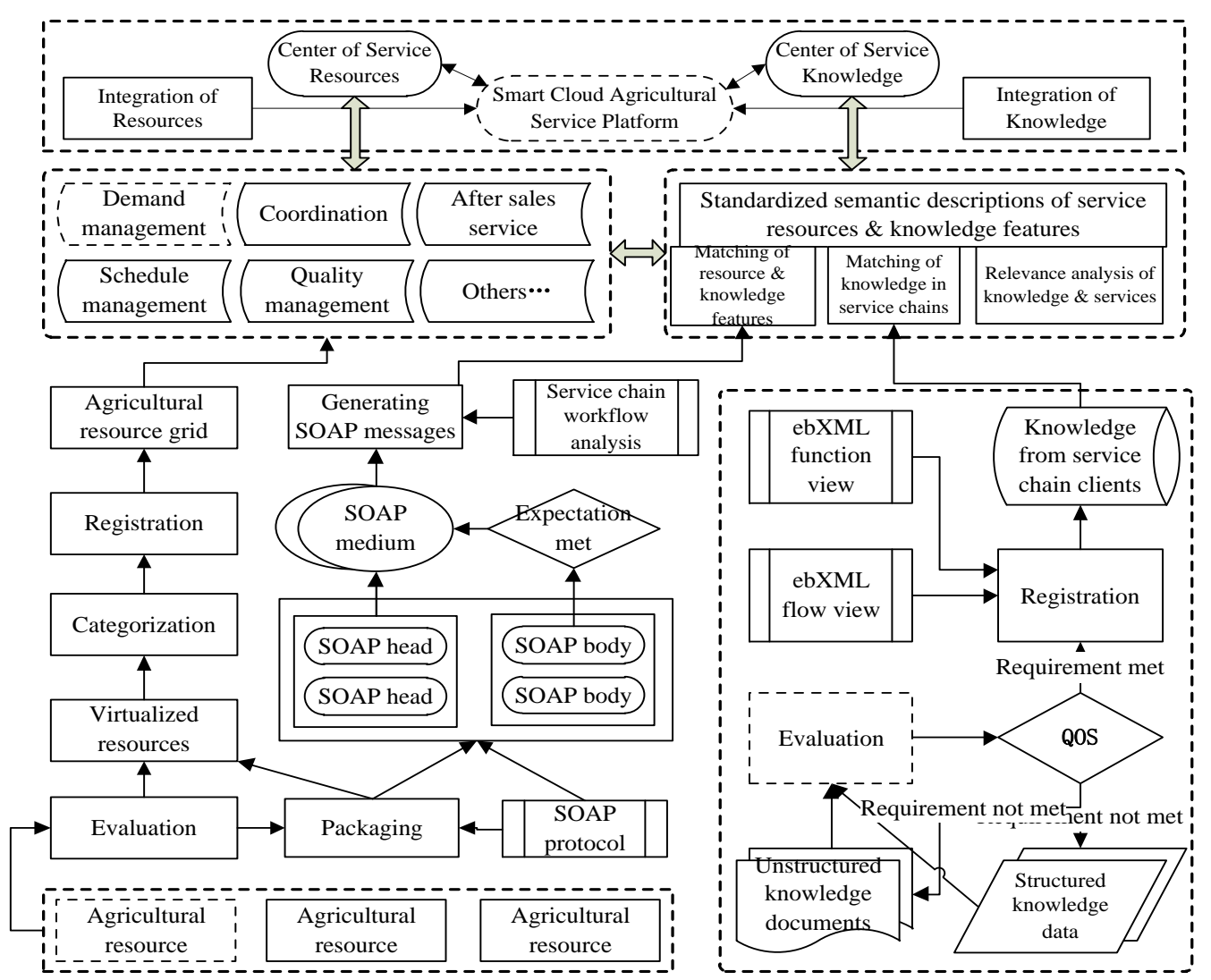

Figure 1. Logical framework of the smart cloud-based agricultural service platform

\subsection{Grid-based integration of service resources and client knowledge}

Agricultural services are packaged into virtualized grid resources, which allow the highly scattered resources to be effectively managed and utilized. This approach is robust and flexible, and mitigates waste from unused resources. The grid-based management maximizes resource use by opening all services and knowledge to be shared between clients.

\subsection{Multiple ways to integrate service resources and client knowledge}

The system can perform identification and semantic interpretation based on user requests. Additionally, it can generatively update its own rules and facts databases by matching subjects to create new rules. The service demands can be compared with historical service records, and similar knowledge can become recommendations for similar services and work processes. ${ }^{[7]}$

\subsection{Connecting client knowledge with service resources}

Knowledge can be extracted from the SOAP information developed from packing service resources. Relevance analysis can be performed to find knowledge with relevant characteristics, which can be pushed to users. The cooperation protocol CPP documents, generated from client uploads, can be used to extract knowledge characteristics, and semantic matching or generative rules can be used to form connections between the service resources and client knowledge.

\subsection{Combination of agricultural service resources and knowledge-sharing portals}

This platform communicates with users via a Web-based interface, using self-containing, self-describing, distributive XML and SOAP-based modules, and each module has a standardized WSDL (Web service description language) description. WSDL descriptions enable users to access services in a standardized manner. In addition to online orders, user management and service data management interfaces (service resource management), the platform also provides user identification, security, and knowledge data services (knowledge management). 


\section{CONCLUSIONS}

As an emerging field with great potential, agricultural IoT is still being developed and tested. While its advantages are obvious, there is presently no firm demand for its applications. The costs of IoT have been too high for traditional agricultural practitioners, and farmers hesitate to invest in it without further proof of its profitability. Some time is still needed before IoT can win over farmers and in some cases, agriculture-related government departments. Other than stronger government support and subsidies, new market-driven commercial models may become the most effective factor in driving the development of agricultural IoT.

\section{ACKNOWLEDGEMENT}

This research was supported by Scientific Research Grant of Guangdong Province (2017A020208007) and Scientific Research Grant of Qingyuan City (2015A005).

\section{REFERENCES}

[1] Li Ning. Applications of IoT in agriculture informatization . Science and Technology Innovation Herald, 2015, 09:92 (in Chinese).

[2] Liu Congfeng, Dai Xiaotong, Chu Chengxiang. Lessons from foreign cases of agricultural IoT. C-Enterprise Management, 2015, 01:62-64 (in Chinese).

[3] Yu Xinrong. Internet of Things: A New Force to Transform Agriculture, Farmers, and Rural Regions . Hefei: Anhui Science \& Technology Press, 2012 (in Chinese).

[4] Han Mingyue (2016). Design of Automatic Control System of IOT (Internet of Things) for Wise Agriculture(in Chinese). Master's Thesis. Harbin Institute of Technology.

[5] Chen Jingmin. Principles, Design \& Application of Data Warehouses . Beijing: China Water \& Power Press, 2004. 153-175 (in Chinese).

[6] Li Youfeng, Wang Zhigang. Research on Dynamic Cloud-based Architecture of Wisdom Agriculture. Computer Technology and Development. 2014, 3(24). 190-193 (in Chinese).

[7] Wang Hu, Gong Chun Qiang, Li Yan. Service-mining Based on the Knowledge and Customer Databases. Proceedings of 6th IEEE/ACIS ICIS 2007, 2007/7: 561-568. 